\title{
On space-time supersymmetry and string duality in nine dimensions
}

\author{
Mohab Abou-Zeid and Hermann Nicolai ${ }^{\mathrm{a}}$ \\ ${ }^{a}$ Max Planck Institut für Gravitationsphysik, Albert Einstein Institut, Am Mühlenberg 1, \\ D-14476 Golm, Germany
}

$N=2$ extended space-time supersymmetry in nine dimensions implies that the BPS states corresponding to momentum and winding of either of the type-II superstrings compactified on $S^{1}$ transform as inequivalent supermultiplets. This fact can be checked using world-sheet techniques, and provides the physical basis for the connection between IIA superstring theory compactified on a circle of radius $R$ and IIB theory compactified on a circle of radius $1 / R$. Aspects of the duality between M-theory compactified on $T^{2}$ and IIB theory compactified on $S^{1}$ follow from the structure of $N=2, D=9$ supergravity. The coupling of $N=2, D=9$ supergravity to the BPS states is also discussed.

In compactifications of the bosonic string theory on a circle of radius $R$, target space duality relates the theory obtained at radius $R$ (we use string units) to the theory compactified at radius $1 / R[1]$. Both the spectrum and the interactions exhibit a symmetry under $R \rightarrow 1 / R$. combined with an interchange of momentum and winding states and a change of sign of the leftmoving oscillators. This symmetry relates large and small distances, so that $R$ can be restricted to the interval $[1, \infty)$ and the theory appears to have a smallest length set by the string scale. At $R=\infty$ the theory decompactifies while at the self-dual point $R=1$ the winding and momentum states acquire equal masses and gauge symmetry enhancement takes place. The heterotic string possesses the same kind of duality symmetry [2]. In the case of the type-II string theories, the situation is different. Duality is not a symmetry, but it is expected to interpolate in a continuous manner between two distinct ten dimensional Lorentz invariant asymptotic theories, viz. the IIA and IIB superstring theories $[3,4]$.

In this contribution, we report on recent work [5] done in collaboration with B. de Wit and D. Lüst. In that paper, a clear physical picture of IIA/B duality was obtained from a consideration of $N=2, D=9$ space-time supersymmetry. The key observation is that, in a compactification of either of the type II theories on a generic spacetime with one compactified coordinate of finite radius, the winding and momentum states carry different space-time quantum numbers: in fact, they constitute inequivalent $N=2, D=9$ supermultiplets at any given compactification radius. It follows that there are no two radii at which the corresponding theories are identical, because their respective spectra are inequivalent; thus the different theories are parametrized by the radius $R$ in the interval $(0, \infty)$. Duality means that two theories that are unrelated in the uncompactified space-time can be viewed as different limits in this 'moduli space' of compactified theories. There is no symmetry enhancement when the masses of momentum and winding states become equal. Also, no conclusion can be drawn as to the existence of a minimal length from IIA/B duality alone. In the following, we summarize the results of [5] which lead to the above picture of IIA/B duality, and comment on further implications of $N=2, D=9$ space-time supersymmetry and supergravity in the context of M theory/IIB string duality [6].

Consider the $D=9$ supersymmetry algebra in an $N=2$ Majorana basis,

$$
\left\{Q_{\alpha}^{i}, Q_{\beta}^{\dagger j}\right\}=\left(\boldsymbol{P}^{0}\right)_{\alpha \beta}+Z^{i j}\left(i \gamma^{0}\right)_{\alpha \beta}
$$

We can decompose the central charge as

$$
Z^{i j}=M\left[b \delta^{i j}+a\left(\cos \theta \sigma_{3}+\sin \theta \sigma_{1}\right)^{i j}\right],
$$

where $M$ is the rest mass of the representation and $\sigma_{1}, \sigma_{2}, \sigma_{3}$ denote the usual Pauli ma- 
trices. The component proportional to $b \mathrm{de}-$ fines an $\mathrm{SO}(2)$ invariant central charge, while the two components proportional to $a$ rotate into each other under the action of the automorphism group $\mathrm{SO}(2)$.

The possible values for $Z^{i j}$ can be obtained from a straightforward reduction of the tendimensional supersymmetry algebra. In the conventions of [5], the ten-dimensional supersymmetry algebra $\{Q, \bar{Q}\}=-i P_{M} \Gamma^{M}$ is converted into the nine-dimensional algebra $\{Q, \bar{Q}\}=-i P_{\mu} \gamma^{\mu}$ $P_{9} \Gamma^{11}$. Hence the sign of the $P_{9}$ term depends on the chirality of the supercharge. The matrix $Z^{i j}$ is proportional to $\left(\sigma_{3}\right)^{i j}$ for IIA theory, where one has supercharges of opposite chirality, and proportional to $\delta^{i j}$ for IIB theory, where the charges have equal chirality.

To exhibit the BPS multiplets in nine dimensions we diagonalize the matrix (2) by an appropriate $\mathrm{SO}(2)$ transformation so that $\theta=0$. In the rest frame the anticommutator (1) decomposes into four eight-dimensional unit matrices, according to the decomposition $\mathbf{8}_{c}+\mathbf{8}_{s}+\mathbf{8}_{c}+\mathbf{8}_{s}$ of the thirty-two supercharges, with coefficients equal to $M$ times $(1+a+b),(1-a-b),(1-a+b)$ and $(1+a-b)$, respectively. Unitarity requires that all of these combinations be non-negative, i.e. $1 \pm a \pm b \geq 0$. We have BPS multiplets whenever one of these coefficients vanishes. We distinguish three basic cases (i. e. the smallest multiplets) according to their decomposition with respect to the rest-frame spin rotation group $\mathrm{SO}(8)$ :

- $a= \pm 1$ and $b=0$ lead to the $2^{8}$ dimensional multiplet $\left(\mathbf{8}_{v}+\mathbf{8}_{s}\right) \times\left(\mathbf{8}_{v}+\mathbf{8}_{c}\right)$. Note that this multiplet contains fermions of mixed chirality. Another characteristic feature is the presence of a $\mathbf{5 6} \boldsymbol{6}_{v}$ spin representation. This is the multiplet that comprises the Kaluza-Klein states of IIA supergravity compactified on $S^{1}$, which are the momentum states of the compactified IIA string. Therefore this particular multiplet will be called the KKA multiplet.

- $a=0$ and $b= \pm 1$ lead to the $2^{8}$ dimensional multiplet $\left(\mathbf{8}_{v}+\mathbf{8}_{c}\right) \times\left(\mathbf{8}_{v}+\right.$ $\mathbf{8}_{c}$ ) (together with the conjugate multiplet). Here the fermions have definite chirality (their partners in the conjugate supermultiplet carry opposite chirality). Observe also the absence of $\mathbf{5 6}_{v}$ states. This supermultiplet comprises the momentum states of the IIB theory and therefore it will be called the KKB multiplet.

- $\pm a \pm b= \pm 1$ lead to multiplets comprising $2^{12}$ states. In string theory, these are mixed states containing both winding and momentum and which have a nonzero oscillator number in order to satisfy the mass-shell condition. Hence they carry masses of the order of the string scale. The smallest multiplet associated with the lowest spins decomposes as $\left(\mathbf{8}_{v}+\mathbf{8}_{c}\right) \times\left(\mathbf{8}_{v}+\mathbf{8}_{c}\right) \times\left(\mathbf{8}_{v}+\mathbf{8}_{s}\right)$. Again there is a conjugate multiplet when changing the signs of $a$ and $b$.

In the literature one often finds the statement that the IIA and the IIB theories become indistinguishable when viewed in a nine-dimensional context, because the $\mathrm{SO}(7)$ decompositions of the IIA and IIB massless multiplets coincide. Although this is true, it is essential to understand that the Kaluza-Klein momentum states for the two theories remain different in nine dimensions: for massive states in nine dimensions, the restframe $\mathrm{SO}(8)$ rotation group coincides with the $\mathrm{SO}(8)$ helicity group for massless states in ten dimensions.

It is furthermore important that the KKA and $\mathrm{KKB}$ multiplets differ not only in their spin decomposition, but also carry inequivalent charges: a KKA supermultiplet carries a nonzero $\mathrm{SO}(2)$ doublet charge while a KKB supermultiplet carries the $\mathrm{SO}(2)$ invariant charge. These charges are mutually exclusive for these multiplets (but not for the 'intermediate' multiplets with $2^{12}$ states). This inequivalence is crucial for duality between the two type-II superstring theories. Indeed, obtaining the other asymptotic theory in the decompactification limit relies on the fact that the winding and momentum modes of a given type-II theory at a given compactification radius constitute inequivalent representations and correspond to different kinds of string states.

To see this, consider the two Majorana supercharges in type-II string theory, which can be rep- 
resented as contour integrals over world-sheet operators:

$$
Q_{\alpha}^{1}=\oint \frac{d z}{2 \pi i} V_{\alpha}(z), \quad Q_{\alpha}^{2}=\oint \frac{d \bar{z}}{2 \pi i} V_{\alpha}(\bar{z})
$$

In the canonical $q=-1 / 2$ ghost picture the two covariant left- and right-moving fermion vertex operators $V_{\alpha}(z)$ and $V_{\alpha}(\vec{z})$ are given by [7] (omitting normal-ordering symbols)

$V_{\alpha(-1 / 2)}(z)=S_{\alpha}(z) \exp \left(-\frac{1}{2} \phi(z)\right)$,

$V_{\alpha(-1 / 2)}(\bar{z})=S_{\alpha}(\bar{z}) \exp \left(-\frac{1}{2} \phi(\bar{z})\right)$,

where $\phi(z)(\phi(\bar{z}))$ is one of the left- (right)moving bosonized superconformal ghosts and $S_{\alpha}(z), S_{\alpha}(\bar{z})$ are the spin field vertex operators in the 16 or $\overline{\mathbf{1 6}}$ chiral spinor representations of $S O(9,1)$. Note that (4) is valid for both IIA and IIB string theory, as we refrain from using dotted and undotted indices to indicate the chirality (it is understood that a projection onto the corresponding chiral subspaces has been made). The ten world-sheet fermions $\psi^{M}(z)$ can be bosonized in terms of five scalars $\vec{\phi}$ as $\exp \left(i \vec{\lambda}_{\mathrm{v}} \cdot \vec{\phi}\right)$, with the $\mathrm{SO}(1,9)$ vector weights $\vec{\lambda}_{\mathrm{v}}=(0, \ldots, \pm 1,0, \ldots)$ (thus $\left.\lambda_{\mathrm{v}}^{2}=1\right)$. The spin field operators $S_{\alpha}(z)$ can be similarly expressed as $\exp \left(i \vec{\lambda}_{\mathrm{s}} \cdot \vec{\phi}(z)\right)$, or $\exp \left(i \vec{\lambda}_{\mathrm{c}} \cdot \vec{\phi}(z)\right)$, where $\vec{\lambda}_{\mathrm{s}}$ and $\vec{\lambda}_{c}$ denote the two $\operatorname{SO}(9,1)$ chiral spinor weights $\left( \pm \frac{1}{2}, \pm \frac{1}{2}, \pm \frac{1}{2}, \pm \frac{1}{2}, \pm \frac{1}{2}\right)$, with an even (odd) number of minus signs for the positive (negative) chirality (and $\lambda_{s}^{2}=\lambda_{c}^{2}=\frac{5}{4}$ ).

The supersymmetry algebra can be computed from the operator products between the vertex operators (4) and their counterparts in the equivalent $q=+1 / 2$ ghost picture [5]. Taking contour integrals and converting to nine-dimensional gamma indices, this yields the following supersymmetry algebra for the IIA/B superstrings in nine space-time dimensions

$$
\begin{aligned}
& \left\{Q^{1}, \bar{Q}^{1}\right\}=-i P_{\mu} \gamma^{\mu}-p_{L} \Gamma^{11}, \\
& \left\{Q^{2}, \bar{Q}^{2}\right\}=-i P_{\mu} \gamma^{\mu}-p_{R} \Gamma^{11}, \\
& \left\{Q^{1}, \bar{Q}^{2}\right\}=0,
\end{aligned}
$$

where $P_{\mu}$ denotes the nine-dimensional momentum and

$p_{L}=\oint \frac{d z}{2 \pi i} i \partial X_{L}^{9}(z)=\frac{m}{R}-n R$,

$$
p_{R}=\oint \frac{d \bar{z}}{2 \pi i} i \bar{\partial} X_{R}^{9}(\bar{z})=\frac{m}{R}+n R
$$

denote the right- and left-moving zero-mode momenta in the compactified direction (with $m$ and $n$ the momentum and winding numbers).

Comparing with the supersymmetry algebra (1), the central charges are seen to be linear combinations of the internal left- and right momenta $p_{L}$ and $p_{R}$. In the IIA and the IIB theory the central charge matrix $Z^{i j}$ takes one of the two alternative forms (up to an overall sign),

$Z^{i j}=\frac{1}{2}\left(p_{L}+p_{R}\right) \delta^{i j}+\frac{1}{2}\left(p_{L}-p_{R}\right)\left(\sigma_{3}\right)^{i j} \mathrm{IIB}$

and

$Z^{i j}=\frac{1}{2}\left(p_{L}-p_{R}\right) \delta^{i j}+\frac{1}{2}\left(p_{L}+p_{R}\right)\left(\sigma_{3}\right)^{i j}$ IIA

This proves that the momentum and winding BPS states constitute inequivalent supermultiplets. The IIA momentum states and the IIB winding states are in the KKA representation, while the IIA winding states and the IIB momentum states are in the $\mathrm{KKB}$ representation. This ensures that the two decompactification limits $R \rightarrow 0$ and $R \rightarrow \infty$ lead to different theories. Moreover, it proves that type-II string compactifications on circles of different radii must be inequivalent. Also, it is clear that no symmetry enhancement will take place when the momentum and the winding states have coinciding masses, as these states are always distinctly different. This is in accord with the fact that no gauge symmetry enhancement is possible in the conformal field theory.

The emergence of different representations for the momentum and winding states can also be understood in terms of the corresponding covariant physical vertex operators for the Kaluza-Klein and winding states in nine dimensions. Consider the Ramond-Ramond operators

$$
\begin{gathered}
\exp \left(i p_{\mu} X_{L}^{\mu}(z)+i p_{L} X_{L}^{9}(z)\right) \\
\times \bar{u}_{L}^{\alpha}(p) S_{\alpha}(z) \exp \left(-\frac{1}{2} \phi(z)\right) \\
\times \exp \left(i p_{\mu} X_{R}^{\mu}(\bar{z})+i p_{R} X_{R}^{9}(\bar{z})\right) \\
\times \bar{u}_{R}^{\beta}(p) S_{\beta}(\bar{z}) \exp \left(-\frac{1}{2} \phi(\bar{z})\right),
\end{gathered}
$$

where the 16-component spinors $u_{L}(p)$ and $u_{R}(p)$ denote the chiral $\mathrm{SO}(1,9)$ spinor polarizations of 
the left- and right-moving states (so that we have implemented a GSO projection [8]) and the $p_{\mu}$ are the values taken by the nine-dimensional momentum operators $P_{\mu}$. Applying the physical state conditions which follow from requiring that the operators (9) commute with the left- and rightmoving BRST operators reduces the number of physical spinor polarizations from 16 to 8 , so that the vertex operators (9) describe $8 \times 8=64$ states for given momentum. When combined with the Neveu-Schwarz sector these states comprise full BPS supermultiplets.

In obtaining the $\mathrm{SO}(8)$ representations in accord with our earlier analysis, it is important to realize that the chirality of the polarization spinors is opposite to that of the corresponding $S_{\alpha}$. Switching the relative chirality, e.g. by changing the chirality of $S_{\alpha}(z)$, and correspondingly of $u_{L}$, can be compensated for by assigning an opposite momentum $p_{L}$ to that state, leaving $p_{R}$ unchanged. This corresponds to interchanging the winding and the momentum numbers $m$ and $n$ in (6), together with the interchange of $R$ with $1 / R$. So the states and the corresponding supermultiplets remain the same; what changes is only the notion of a momentum and a winding statc. The mass-shell condition then tells us that $p_{L}= \pm p_{R}$, and depending on this sign, we get either the same or different $\mathrm{SO}(8)$ representations from the physical state condition. Thus winding and momentum states indeed constitute inequivalent supermultiplets. In the decompactification limits $R \rightarrow 0$ and $R \rightarrow \infty$, one is left with inequivalent supermultiplets as the mass of one supermultiplet vanishes and that of its inequivalent counterpart is pushed to infinity.

The result that the momentum and winding states are in different supermultiplets can be independently derived from $N=2$ supergravity in $D=9$ dimensions (together with some basic input from string theory). This theory is discussed in [9]; here we exhibit the coupling of the ninedimensional gauge fields to the BPS states discussed above. Recall that the scalar sector is governed by an $\mathrm{SL}(2, \mathbb{R}) / \mathrm{SO}(2)$ non-linear $\sigma$-model, which therefore exhibits an invariance under a nonlinearly realized $\mathrm{SL}(2, \mathbb{R})$. In addition there is an invariance under $\mathrm{SO}(1,1)$, which can be sys- tematically understood from combining ordinary dimensional analysis with scale transformations on the compactified coordinate [10]. From the IIB supergravity perspective, the $\mathrm{SL}(2, \mathbb{R})$ originates from the $\mathrm{SL}(2, \mathbb{R}) / \mathrm{SO}(2)$ coset structure and the $\operatorname{SL}(2, \mathbb{R})$ symmetry which are already present in ten dimensions [11]. From the perspective of eleven-dimensional supergravity [12], on the other hand, these are just the 'hidden' symmetries obtained by reducing the theory from eleven to nine dimensions on the torus $T^{2}$. In this reduction, the diffeomorphism symmetry in the compactified dimensions is 'frozen' to a rigid $\mathrm{GL}(2, \mathbb{R})=\mathrm{SL}(2, \mathbb{R}) \times \mathrm{SO}(1,1)$ symmetry. Similarly, the full Lorentz symmetry in eleven dimensions is reduced to $\mathrm{SO}(1,8) \times \mathrm{SO}(2) \subset \mathrm{SO}(1,10)$, where $\mathrm{SO}(2)$ is converted into the R-symmetry corresponding to the automorphism group of the nine-dimensional $N=2$ superalgebra (1). We denote the bosonic fields of eleven-dimensional supergravity by $\hat{G}_{\hat{M} \hat{N}}$ and $\hat{A}_{\hat{M} \hat{N} \hat{P}}$. The bosonic fields of IIA supergravity are denoted by $G_{M N}$, $C_{M}, C_{M N}, C_{M N P}$ and $\phi$, and those of IIB supergravity by $G_{M N}, A_{M N}^{\alpha}, \phi^{\alpha}$ and $A_{M N P Q}$. Here the index $\alpha$ is associated with $\operatorname{SL}(2, \mathbb{R})$. The fields of $N=2$ nine-dimensional supergravity are the metric $g_{\mu \nu}$, three scalars $\sigma$ and $\phi^{\alpha}$, three abelian gauge fields $B_{\mu}$ and $A_{\mu}^{\alpha}$, two antisymmetric tensors $A_{\mu \nu}^{\alpha}$ and a three-rank antisymmetric gauge field $A_{\mu \nu \rho}$. We use the Einstein frame, so that the metric is invariant under $\operatorname{SO}(1,1)$. The scalar fields $\phi^{\alpha}$ characterize the coset representative of $\mathrm{SL}(2, \mathbb{R}) / \mathrm{SO}(2)$. They satisfy a constraint $\phi^{\alpha} \phi_{\alpha}=1$ and are subject to local $S O(2)$ transformations, so that they correspond to one complex field. The scalar $\operatorname{cxp}(\sigma)$ will be defined as $G_{99}$, the IIB metric in the compactified dimension. The determinant of the eleven-dimensional metric in the two compactified directions is then equal to $\exp \left(-\frac{4}{3} \sigma\right)$.

Now consider the three abelian vector gauge fields in the nine-dimensional theory, which decompose into a singlet and a doublet under $\mathrm{SL}(2, \mathbb{R})$. Note that their origin is rather different when viewed from the IIA and from the IIB side. The singlet field is the graviphoton from the IIB side, so it must couple to the IIB momentum states. The doublet fields originate from the 
IIB doublet of tensor fields, so they couple to the IIB winding states. It thus follows that the IIB momentum states constitute KKB states (by definition) whereas the IIB winding states constitute KKA multiplets. The second KKA charge can only be understood beyond string perturbation theory; the degeneracy in the winding states is due to winding of fundamental and D-strings [13]. The pattern is the same, but complementary on the IIA side. Here the momentum states carry the doublet charges, so they constitute (again by definition) KKA multiplets. Accordingly, the two graviphotons originating from eleven dimensions transform as an $\mathrm{SL}(2, \mathbb{R})$ doublet. The degeneracy in the momentum states can thus be understood from eleven-dimensional supergravity, as the doublet charges find their origin in the $T^{2}$ on which the theory is compactified. The winding states couple to the singlet field, which originates from the IIA tensor field. Hence the IIA winding states constitute KKB multiplets. Alternatively these states can be understood as eleven dimensional supermembranes [14] wrapped around the M-theory torus [6], as these constitute the same supermultiplets.

To see this last point, consider the supersymmetry algebra in eleven dimensions with a membrane charge,

$$
\{Q, \bar{Q}\}=-i P_{\hat{M}} \Gamma^{\hat{M}}+\frac{1}{2} i Z_{\hat{M} \hat{N}} \Gamma^{\hat{M} \hat{N}}
$$

where we have eleven-dimensional momenta $P_{\dot{M}}$, two-brane charges $Z_{\hat{M} \hat{N}}$ and 32-component spinor charges. The $Z_{\hat{M} \hat{N}}$ can describe the winding of a fundamental supermembrane over some compact space [15]. Upon reducing the algebra (10) to nine dimensions and assuming that $Z_{\hat{M} \hat{N}}$ takes only values in the two internal dimensions labeled by $\hat{M}=9,10$, we obtain for the central-charge matrix $Z^{i j}$,

$Z^{i j}=Z_{910} \delta^{i j}-\left(P_{9} \sigma_{3}-P_{10} \sigma_{1}\right)^{i j}$.

Thus the BPS states associated with a membrane wrapped around $T^{2}$ in eleven dimensions constitute KKB multiplets. Observe that this is crucial for the duality between M-theory and IIB theory noted in [6].

From the result (11), we can also deduce the general BPS mass formula,

$$
M=\sqrt{P_{9}^{2}+P_{10}^{2}}+\left|Z_{910}\right| .
$$

For a supermembrane wrapped around a torus with modular parameter $\tau \equiv \tau_{1}+i \tau_{2}$ and area $A$, the BPS mass formula follows directly from (12) and reads (in eleven-dimensional Planck units)

$M=\frac{1}{\sqrt{A \tau_{2}}}\left|q_{1}-\tau q_{2}\right|+A T_{\mathrm{m}}|p|$,

where $T_{\mathrm{m}}$ denotes the supermembrane tension, $q_{1,2}$ label the momentum modes on the torus and $p$ is the number of times the membrane is wrapped (including orientation) over the torus. This formula agrees with the one previously derived in [16] on the basis of a semi-classical approximation.

The above considerations suggest the construction of a nine-dimensional field theory consisting of $N=2$ supergravity coupled to an infinite tower of BPS supermultiplets with a two-dimensional charge lattice $\left(q_{1}, q_{2}\right)$ for the KKA states and a one-dimensional lattice of charges $p$ for the KKB states. This theory encompasses both elevendimensional supergravity (compactified on $T^{2}$ ) and IIB supergravity (compactified on $S^{1}$ ). The usual T-duality is trivial for this theory. It is not associated with any symmetry and only amounts to certain field redefinitions. The theory is free from inconsistencies in each of these sectors separately and it is an interesting question whether such a 'dichotomic' field theory could be (classically) consistent to all orders. In low orders of perturbation theory, its short-distance behaviour should be relatively mild as it can be viewed as a combination of known supergravity theories. Of course, this is not truly an effective field theory as the masses of the various states will never be light simultaneously with respect to the string scale. The theory is manifestly invariant under under $\mathrm{SL}(2, \mathbb{Z})$. The latter is the integer-valued subgroup of $\operatorname{SL}(2, \mathbb{R})$ that leaves the charge lattice of the KKA states invariant. There is a formulation in which the $\mathrm{SL}(2, \mathbb{R})$ is linearly realized, also in the presence of the BPS states. In that case the massive fields transform only under the local (composite) SO(2) and not directly under $\operatorname{SL}(2, \mathbb{R})$. However, the KKA fields have a 
minimal coupling with respect to $q_{\alpha} A_{\mu}^{\alpha}$, which, in order to remain invariant under the integervalued subgroup, requires the charges to transform covariantly under this subgroup. The KKB fields have a minimal coupling to $p B_{\mu}$, which is $\mathrm{SL}(2, \mathbb{R})$ invariant.

It should be clear that the theory will exhibit ten- or eleven-dimensional Lorentz invariance only in certain limits. For the KKA states with charges $q_{\alpha}$, and KKB states with charge $p$, respectively, the BPS mass formula in the ninedimensional Einstein frame is given by

$M=m_{\mathrm{KKA}}\left|q_{\alpha} \phi^{\alpha}\right|+m_{\mathrm{KKB}}|p|$,

where $m_{\mathrm{KKA}}$ and $m_{\mathrm{KKB}}$ denote two different mass scales, whose product is inversely proportional to $\alpha^{\prime}$. Here we made use of the fact that the mass should be $\operatorname{SL}(2, \mathbb{Z})$ invariant in the Einstein frame.

The formula (14) can now be interpreted in two different ways. From the perspective of IIA string theory, one of the $q_{\alpha}$ is the IIA Kaluza-Klein momentum number, while the other is the D0 charge; as is well known, the mass of the D0 branes is inversely proportional to the IIA string coupling constant [13]. Then $p$ is the IIA winding number. Conversely, from the IIB perspective, $q_{1}$ and $q_{2}$ are the winding numbers of the elementary string and of the solitonic D1 string (which corresponds to a D0 brane in the IIA description). Now the $\mathrm{SL}(2, \mathbb{Z})$ is a strong-weak coupling duality, as it interchanges the elementary strings with the D1 strings. The modular parameter associated with the fields $\phi^{\alpha}$ is the IIB dilaton which contains the IIB string coupling constant. From this perspective the integral charge lattice follows from a Dirac-type quantization condition. The integer $p$ is just the IIB Kaluza-Klein momentum number.

Although (14) does not include the contributions from the 'intermediate' BPS multiplets which carry both the doublet charges $q_{\alpha}$ and the singlet charge $p$, the analysis can be straightforwardly generalised to accommodate them. An interesting question is whether such supermultiplets play a role in certain threshold calculations in toroidally compactified M-theory to which only BPS multiplets are expected to contribute (see e. g. $[17,18]$ for reviews). We hope to return to this issue elsewhere.

\section{ACKNOWLEDGEMENTS}

We would like to thank our co-authors Bernard de Wit and Dieter Lüst for fruitful collaboration and discussions.

\section{REFERENCES}

1. K. Kikkawa and M. Yamasaki, Phys. Lett. B149 (1984) 357; N. Sakai and I. Senda, Prog. Theor. Phys. 75 (1986) 692; Erratum-ibid 77 (1987) 773.

2. P. Ginsparg, Phys. Rev. D35 (1987) 648.

3. M. Dine, P. Huet and N. Seiberg, Nucl. Phys. B322 (1989) 301.

4. J. Dai, R.G. Leigh and J. Polchinski, Mod. Phys. Lett. A4 (1989) 2073.

5. M. Abou-Zeid, B. de Wit, D. Lüst and H. Nicolai, Space-Time Supersymmetry, IIA/B Duality and $\mathrm{M}$ Theory, hep-th/9908169, to appear in Phys. Lett. B.

6. J.H. Schwarz, Phys. Lett. B367 (1996) 97, [hep-th/9510086]; P. S. Aspinwall, Nucl. Phys. Proc. Suppl. 46 (1996) 30 [hepth/9508154].

7. D. Friedan, E. Martinec and S. Shenker, Nucl. Phys. B271 (1986) 93.

8. F. Gliozzi, J. Scherk and D. Olive, Phys. Lett. B65 (1976) 282; Nucl. Phys. B122 (1977) 253.

9. E. Bergshoeff, C. Hull and T. Ortin, Nucl. Phys. B451 (1995) 547 [hep-th/9504081].

10. B. de Wit and J. Louis, Supersymmetry and Dualities in Various Dimensions, hepth/9801132.

11. J.H. Schwarz, Nucl. Phys. B226 (1983) 269.

12. E. Cremmer, B. Julia and J. Scherk, Phys. Lett. B76 (1978) 409.

13. J. Polchinski, Phys. Rev. Lett. 75 (1995) 184 [hep-th/9510017].

14. E. Bergshoeff, E. Sezgin and P.K. Townsend, Phys. Lett. 189B (1987) 75.

15. B. de Wit, K. Peeters and J. Plefka, Phys. Lett. B409 (1997) 117 [hep-th/9705225].

16. J. Russo and A. Tseytlin, Nucl. Phys. B 490 (1997) 121 [hep-th/9611047]. 
17. M. B. Green, Nucl. Phys. Proc. Suppl. 68 (1998) 242 [hep-th/9712195].

18. E. Kiritsis, Duality and Instantons in String Theory, hep-th/9906018. 